\title{
Determinants of Preeclampsia Among Pregnant Mothers Attending Antenatal Care (ANC) and Delivery Service in Gedeo Zone, Southern Ethiopia: Case Control-Study
}

This article was published in the following Dove Press journal:

International Journal of Women's Health

\author{
Moges Mareg (D) \\ Alemayehu Molla iD $^{2}$ \\ Selamawit Dires' \\ Zerihun Berhanu Mamo (D) \\ Brhane Hagos $\mathbb{D}^{3}$
}

'Reproductive Health Department, School of Public Health, Dilla University, College of Health Science and Medicine, Dilla, Ethiopia; ${ }^{2}$ Department of Psychiatry, College of Health Science and Medicine, Dilla University, Dilla, Ethiopia; ${ }^{3}$ Department of Midwifery, College of Health Science and Medicine, Dilla University, Dilla, Ethiopia
Correspondence: Moges Mareg Reproductive Health Department, College of Health Science and Medicine, School of Public Health, Dilla University, Dilla, Ethiopia

Tel +251923527030

Email metanmann@gmail.com
Background: The maternal morbidity and mortality related to preeclampsia are increasing in developing countries; figures have been estimated to be between $1.8 \%$ and $16.7 \%$, including in Ethiopia. However, there is limited research regarding the determinants of preeclampsia in Gedeo Zone.

Objective: The aim of the study was to determine the predictors of preeclampsia among pregnant mothers attending ANC and delivery services in southern Ethiopia.

Methods: A facility-based unmatched case-control study was conducted in Gedeo Zone. Pregnant mothers attending ANC and delivery service were selected consecutively until the allotted 243 (162 controls and 81 cases) sample size was fulfilled. The collected data were checked for completeness and entered into Epi-data software version 3.1, and exported to statistical package for social science (SPSS) version 20 for analysis. Multivariable logistic regression was employed and a $P$-value of less than 0.05 with a $95 \%$ confidence interval was used to declare the significant association of the independent variables and the outcome variable. Results: A total of 240 mothers, 80 (33.3\%) of cases and 160 (66.70\%) of controls, responded, with a response rate of $98.76 \%$. The mean age of the participants among both groups was 27.40 with a standard deviation of \pm 5.02 . Attending education [adjusted odd ratio (AOR) $=0.49 ; 95 \%$ CI $(0.006,0.398)]$, being a house wife $[\mathrm{AOR}=13 ; 95 \% \mathrm{CI}(1.260,140.15)]$, an age range of 20-34 years $[\mathrm{AOR}=0.071 ; 95 \% \mathrm{CI}(0.015,0.32)]$, a family history of diabetes mellitus [AOR = $0.28 .2 ; 95 \% \mathrm{CI}(0.081,0.985)]$, a family history of hypertension $[\mathrm{AOR}=0.124 ; 95 \% \mathrm{CI}(0.047$, $0.325)]$, did not eat fruit during pregnancy $[\mathrm{AOR}=3.355 ; 95 \% \mathrm{CI}(1.112,10.126)]$, and a maternal history of preeclampsia $[\mathrm{AOR}=0.162 ; 95 \% \mathrm{CI}(0.041,0.640)]$ were found to be variables significantly associated among mothers with preeclampsia.

Conclusion: The determinant factors for preeclampsia were being a housewife, having a history of hypertension, diabetes mellitus, and preeclampsia among family members. Strengthening early detection and prevention of predictors, improvement of protective factors, and further follow-up study were recommended.

Keywords: antenatal care, delivery, preeclampsia, pregnant mothers, southern Ethiopia

\section{Introduction}

According to the International Classification of Diseases, tenth revision (ICD-10), preeclampsia is defined as a diastolic blood pressure of at least $90 \mathrm{mmHg}$ on 2 or more successive occurrences of at least 4 hours apart or a diastolic blood pressure of at least $110 \mathrm{mmHg}$ on any 1 occurrence, plus proteinuria of at least $300 \mathrm{mg}$ per 
24 hour or $>1+$ on a urine dipstick, ${ }^{1,2}$ after the 20 th week of gestation in a previously normotensive and non-proteinuric pregnant woman. ${ }^{2}$

The etiology of preeclampsia, also known as pregnancy-induced hypertension (PIH), is unknown. ${ }^{3}$ Though the cause of preeclampsia is unfamiliar, there are definite determinant risk factors related to the condition. The factors that have been proposed to influence the risk of preeclampsia among mothers comprise diabetes, renal disease, obesity, multiple pregnancy, prime-parity, age above 30 years, personal or family history of preeclampsia, and chronic hypertension. Preeclampsia can be quite serious as it can lead to various complications both for the mother and the baby. ${ }^{3}$

Preeclampsia is a foremost contributor to maternal and newborn morbidity and mortality. ${ }^{4}$ Hereditary, immunological, ecological, and vascular-mediated causes are all supposed to play a substantial role in the intensification of preeclampsia, ${ }^{5,6}$ and numerous risk factors have been identified. Among those, null-parity, previous preeclampsia, high maternal weight, hypertension, diabetes, and twin pregnancy have shown a consistent association with increased risk. $^{6-8}$

Globally, greater than $80 \%$ of maternal mortalities are due to 5 direct causes: hemorrhage, infection, unsafe abortion, pulmonary embolism, and hypertensive disorder of pregnancy (HDP). ${ }^{7}$ Around 289,000 mothers pass away worldwide from pregnancy-related reasons in 2013. Of these, $99 \%$ of deaths occur in developing countries. SubSaharan Africa accounts for about $56 \%$ of all maternal mortality. A woman's lifetime risk of dying of pregnancy-related problems is 14 times higher in developing countries than in developed countries. ${ }^{8}$

The World Health Organization (WHO) estimates that each year between 50,000 and 75,000 mothers die due to this disorder globally, as PIH is accountable for $12 \%$ of maternal mortality worldwide. ${ }^{9}$ The influence of the disorder is more severe in less developed countries. The prevalence of preeclampsia in less developed countries has been predicted between $1.8 \%$ and $16.7 \%{ }^{10}$ with at least $16 \%$ of maternal mortality resulting from preeclampsia. ${ }^{11}$

According to the 2016 Ethiopian Demographic and Health Survey (EDHS), an estimated 412 mothers per 100,000 live births died due to gestation and related causes. $^{10}$ Underdeveloped countries including Ethiopia face worse complications of the problem than developed ones. Poor pregnancy outcomes that are related to lack of
ANC follow-up and delayed recognition and intervention in the affected mothers have worsened the situation. ${ }^{11}$

Although providing due attention to such causes of maternal death could speed the reduction of maternal death, determinants of preeclampsia have not been well recognized in Gedeo Zone. Therefore, this study was aimed at identifying the determinant factors of preeclampsia. Documentation of potential determinant factors for the increment of PE helps to prevent an increasing incidence of PE. The study will also provide a source of important information for scholars for further research on the field, decision-makers, and policy and strategic designers.

\section{Materials and Methods \\ Study Setting and Period}

An unmatched case-control study was conducted in Gedeo Zone, in 3 public hospitals from January 1 to February 30, 2019 to determine the predictors of preeclampsia among pregnant mothers. Gedeo is one of the 11 zones found in Southern Nations, Nationalities and Peoples Region (SNNPR) with the administrative center of Dilla town located $360 \mathrm{~km}$ from Addis Ababa (the capital city of Ethiopia). According to the Gedeo Zone health department, the estimated number of mothers of reproductive age (15-49) is 256,487 and the estimated number of deliveries is 3286. In Gedeo Zone, the skilled delivery rate is $57.43 \%$. The zone has one referral hospital, three district hospitals, 35 health centers, and 146 health posts. In addition, there are 9 nongovernmental organizations (NGOs) providing health service for the community.

\section{Sample Size Calculation and Sampling Technique}

The sample size was calculated by using EPI INFO version 7, after considering the following issues: primigravida as a risk factor with a lowest odds ratios of $2.68 \%$ and $37.37 \%$ among controls, ${ }^{12} 95 \%$ confidence interval, 5\% margin of error, and $80 \%$ power. The calculated sample size was 220 , and after adding a $10 \%$ non-response rate, the final sample size was 243 ( 81 cases and 162 controls).

All participants admitted for ANC and delivery services who fulfilled the inclusion criteria in the 3 hospitals were consecutively included until the allotted sample size was achieved. The study used an incidence density sampling approach, which is intended to produce a set of controls, to select two control mothers who were admitted for delivery services in the same facilities for each case. 
The sample size was distributed between the 3 hospitals proportionally to their delivery and ANC load.

\section{Data Collection Instrument and Personnel}

An interviewer-administered pretested questionnaire was used to collect the data. The tool was prepared by reviewing different literature, ${ }^{13-15}$ By using this tool we collected data related to socio-demographic variables, monthly income variable, obstetric and reproductive factors, family history of chronic diseases, and dietary and lifestyle variables. To obtain clinical laboratory results, the respondents' medical charts were reviewed. Three diploma midwives were hired for data collection. Two degreelevel nurses supervised the data collection process and the investigators coordinated them. The supervisors checked the uniformity, clearness, and completeness of the collected data on a daily basis.

\section{Data Quality Management}

To assure the quality of data, the questionaire was translated from English to the local language, Gediuffa/ Amharic, and then back to English; to ensure its uniformity, a pre-test was conducted (in Gedeb district hospital on 5\% (13) of samples); training was given to supervisors and data collectors, and strict day-to-day follow-up and correction was done.

\section{Data Processing and Analysis}

Data were entered into EPI Data version 3.1 and exported to statistical package for social science (SPSS) version 20 for analysis. Descriptive data were summarized using tables. Binary logistic regression was used to identify factors associated with the outcome variable. Variables with a $P$-value of less than 0.25 in bivariate regression were considered for multivariable logistic regression. Finally, $P$-value $\leq 0.05$ in multivariate logistic regression was considered as statistically significant and the strength of associations was determined using adjusted odds ratio (AOR) at corresponding 95\% confidence interval.

\section{Result}

\section{Socio-Demographic Characteristics of Participants}

A total of 240 mothers, 80 (33.3\%) cases and $160(66.70 \%)$ controls, participated in this study with a response rate of $98.76 \%$. The mean ages of the participants in both groups were 27.40 with a standard deviation of \pm 5.02 . More than half of the participants were between the ages of 20 and 34 years. Most of the participants were married 223 (92.9\%). Regarding education, 11 (13.8\%) of cases and 42 (26.2\%) of controls had attended college and above (Table 1).

\section{Obstetric and Reproductive Health- Related Factors}

Regarding the number of pregnancies, more than half of the cases, 51 (63.8\%), and $86(54.4 \%)$ of controls had had 2-4 pregnancies. Regarding the ANC service utilization during pregnancies, 73 (91.2\%) of cases and 150 (93.8\%) of controls had ANC follow-up. Eighteen $(24.7 \%)$ of the cases and $55(36.7 \%)$ of the controls had more than four ANC visits during the current pregnancy. The history of abortion was $12(15.0 \%)$ and 19 $(11.9 \%)$ among cases and controls, respectively. Eight $(66.7 \%)$ of the cases had had an abortion once, 3 $(25.0 \%)$ twice, and $1(8.3 \%)$ more than twice; 18 (94.7\%) of controls had a history of one abortion, and $1(5.3 \%)$ had had two abortions (Table 2).

\section{Medical Illness Related Factors}

Regarding the medical illness-related factors, the family history of diabetes mellitus was $11(13.8 \%)$ and $6(3.8 \%)$ in cases and controls, respectively. The family history of hypertension was also reported at $26(32.5 \%)$ in cases and $8(5.0 \%)$ in controls. Among those who had a family history of hypertension, 5 (19.2\%) of cases had a paternal history, $18(69.2 \%)$ were maternal, $2(7.7 \%)$ reported hypertension in sisters, and $1(3.8 \%)$ in a brother; among controls, 2 (25.0\%) instances were paternal, 5 (62.5\%) were maternal, and $1(12.5 \%)$ was in a sister. Regarding the factors related to participant's maternal history of preeclampsia, $16(20 \%)$ of cases and $4(2.5 \%)$ of controls had a history of maternal preeclampsia.

\section{Dietary and Lifestyle Related Factors}

The body mass index (BMI) of $5(6.2 \%)$ cases and 17 $(10.6 \%)$ controls was less than 18.5, the BMI of 54 $(67.5 \%)$ cases and $103(64.4 \%)$ controls was $18.5-$ 24.5, BMI of $19(23.8 \%)$ cases and $35(21.9 \%)$ controls was 24.5- 29.9, and BMI of $2(2.5 \%)$ cases and 5 (3.1\%) controls was above 30 . The mid upper arm circumference (MUAC) of $11(13.8 \%)$ cases and 34 $(21.2 \%)$ controls was less than $23 \mathrm{~cm}$, MUAC of 61 $(76.2 \%)$ cases and $104(65.0 \%)$ controls was 23- 
Table I Socio-Demographic Characteristics of Participants Who Attend ANC and Delivery Services at Gedeo Zone Hospitals, Southern Ethiopia, 2019 ( $n=240$ (80 Cases, 160 Controls))

\begin{tabular}{|c|c|c|}
\hline \multirow[t]{2}{*}{ Variables } & \multicolumn{2}{|c|}{ Study subject(240) } \\
\hline & $\begin{array}{l}80 \text { Cases }= \\
\text { number }(\%)\end{array}$ & $\begin{array}{l}160 \text { Controls = } \\
\text { number (\%) }\end{array}$ \\
\hline \multicolumn{3}{|l|}{ Age } \\
\hline$<20$ years & $10(12.5)$ & $15(9.5)$ \\
\hline 20-34 years & $62(77.2)$ & $125(79.1)$ \\
\hline$>35$ years & $8(10.0)$ & $18(11.4)$ \\
\hline Mean (SD) & $27.51(5.22)$ & $27.36(4.93)$ \\
\hline \multicolumn{3}{|l|}{ Ethnicity } \\
\hline Gedeo & $45(56.2)$ & $101(63.1)$ \\
\hline Amahara & $9(11.2)$ & $19(11.9)$ \\
\hline Oromo & $7(8.8)$ & $22(13.8)$ \\
\hline Gurage & $10(12.5)$ & $6(3.8)$ \\
\hline Other $^{\mathrm{a}}$ & $9(\mid I .2)$ & $12(7.5)$ \\
\hline \multicolumn{3}{|l|}{ Religion } \\
\hline Protestant & $47(58.8)$ & $108(67.5)$ \\
\hline Orthodox & $12(15.0)$ & $13(8.1)$ \\
\hline Muslim & $2 I(26.2)$ & $39(24.4)$ \\
\hline \multicolumn{3}{|l|}{ Marital status } \\
\hline Single & $3(3.8)$ & $7(4.4)$ \\
\hline Married & $72(90.9)$ & $151(94.4)$ \\
\hline Divorced & $5(6.2)$ & $2(1.2)$ \\
\hline \multicolumn{3}{|l|}{ Educational status } \\
\hline Cannot read and write & $28(35.0)$ & $46(28.8)$ \\
\hline Can read and write & $28(35.0)$ & $30(18.8)$ \\
\hline Primary education & $5(6.2)$ & $23(14.4)$ \\
\hline Secondary education & $8(10.0)$ & $19(11.9)$ \\
\hline College and above & $\mathrm{II}(\mathrm{I} 3.8)$ & $42(26.2)$ \\
\hline \multicolumn{3}{|l|}{ Residences } \\
\hline Urban & $30(37.5)$ & $47(29.4)$ \\
\hline Rural & $50(62.5)$ & $113(70.6)$ \\
\hline \multicolumn{3}{|l|}{ Occupation } \\
\hline Government employee & $20(25.0)$ & $47(29.4)$ \\
\hline Private & $9(I I .2)$ & $22(13.8)$ \\
\hline Housewife & $30(37.5)$ & $60(37.5)$ \\
\hline Merchant & $16(20.0)$ & $25(15.6)$ \\
\hline Other* & $5(6.2)$ & $6(3.8)$ \\
\hline \multicolumn{3}{|l|}{ Monthly Income } \\
\hline $570-1140$ & $5(9.3)$ & I4(II.4) \\
\hline$\geq 1140$ & 49(90.7) & $109(88.6)$ \\
\hline
\end{tabular}

Notes: *Other: daily laborer, student; ${ }^{\mathrm{a} O t h e r: ~ S i d a m a, ~ W o l i t a, ~ S i l t e . ~}$

$24.9 \mathrm{~cm}$, MUAC of $8(10.0 \%)$ cases and $22(13.8 \%)$ controls was above $25 \mathrm{~cm}$. Seventy-one $(88.8 \%)$ cases and $150(93.8 \%)$ controls ate fruit during pregnancy.
Table 2 Obstetric and Reproductive Health Characteristics of Participants Who Attend ANC and Delivery Services at Gedeo Zone Hospitals, Southern Ethiopia, 2019 ( $n=240$ (80 Cases, 160 Controls))

\begin{tabular}{|c|c|c|}
\hline \multirow[t]{2}{*}{ Variables } & \multicolumn{2}{|c|}{ Study subject(240) } \\
\hline & $\begin{array}{l}80 \text { Cases = } \\
\text { number(\%) }\end{array}$ & $\begin{array}{l}160 \text { Controls = } \\
\text { number (\%) }\end{array}$ \\
\hline \multicolumn{3}{|l|}{$\begin{array}{l}\text { Age at first menses (in } \\
\text { years) }\end{array}$} \\
\hline $10-13$ & $23(28.8)$ & $57(24.1)$ \\
\hline $14-16$ & $49(61.2)$ & $160(67.5)$ \\
\hline $17-19$ & $8(10.0)$ & $20(8.4)$ \\
\hline \multicolumn{3}{|l|}{$\begin{array}{l}\text { Age at first pregnancy } \\
\text { (in years) }\end{array}$} \\
\hline$<20$ & $43(53.8)$ & $82(51.9)$ \\
\hline $20-34$ & $37(46.2)$ & $76(48.1)$ \\
\hline \multicolumn{3}{|l|}{$\begin{array}{l}\text { Pregnancy interval(in } \\
\text { years) }\end{array}$} \\
\hline One & $3(4.8)$ & $4(3.8)$ \\
\hline Two & $26(4 I .3)$ & $43(4 I .0)$ \\
\hline$>2$ & $34(54.0)$ & $58(55.2)$ \\
\hline Number of live births & & $62(59.0)$ \\
\hline One & $35(58.3)$ & $22(21.0)$ \\
\hline $2-4$ & $12(20.0)$ & $2 I(20.0)$ \\
\hline$\geq 5$ & $13(21.7)$ & \\
\hline \multicolumn{3}{|l|}{$\begin{array}{l}\text { The number of ANC } \\
\text { follow-ups }\end{array}$} \\
\hline One time & $4(5.5)$ & $8(5.3)$ \\
\hline Two times & $19(26.0)$ & $28(18.7)$ \\
\hline Three times & $32(43.8)$ & $59(39.3)$ \\
\hline Four and above & $18(24.7)$ & $55(36.7)$ \\
\hline \multicolumn{3}{|l|}{ Pregnancy multiplicity } \\
\hline Single & $77(96.2)$ & I57(98.1) \\
\hline Twin & $3(3.8)$ & $3(1.9)$ \\
\hline \multicolumn{3}{|l|}{$\begin{array}{l}\text { Type of contraceptive } \\
\text { method used }\end{array}$} \\
\hline Pills & $4(6.2)$ & $13(10.9)$ \\
\hline Injectable & $36(56.2)$ & $67(56.3)$ \\
\hline Implant & $23(35.9)$ & $39(32.8)$ \\
\hline IUCD & $\mathrm{I}(\mathrm{I} .6)$ & $0(0.00)$ \\
\hline \multicolumn{3}{|l|}{ Blood group } \\
\hline A & $25(31.2)$ & $55(34.4)$ \\
\hline B & $25(31.2)$ & $5 I(31.9)$ \\
\hline$A B$ & $12(\mid 5.0)$ & $2 I(I 3 . I)$ \\
\hline $\mathrm{O}$ & $18(22.6)$ & $33(20.6)$ \\
\hline \multicolumn{3}{|l|}{ Hemoglobin level } \\
\hline Less than II & $22(28.6)$ & $46(29.7)$ \\
\hline Greater than II & $55(71.4)$ & $109(70.3)$ \\
\hline
\end{tabular}


Table 3 Dietary and Lifestyle Characteristics of Participants Who Attend ANC and Delivery Services at Gedeo Zone Hospitals, Southern Ethiopia, 2019

\begin{tabular}{|c|c|c|}
\hline \multirow[t]{2}{*}{ Variables } & \multicolumn{2}{|c|}{ Study subject(240) } \\
\hline & $\begin{array}{l}80 \text { Cases }= \\
\text { No. }(\%)\end{array}$ & $\begin{array}{l}160 \text { Controls = } \\
\text { No. }(\%)\end{array}$ \\
\hline \multicolumn{3}{|l|}{$\begin{array}{l}\text { How many times you ate } \\
\text { fruit }\end{array}$} \\
\hline Daily/week & $9(12.7)$ & $33(22.0)$ \\
\hline 2 to 3 days/week & $59(83.1)$ & III (74.0) \\
\hline Less than 2 times/week & $3(4.2)$ & $6(4.0)$ \\
\hline \multicolumn{3}{|l|}{$\begin{array}{l}\text { Ate vegetables during } \\
\text { pregnancy }\end{array}$} \\
\hline Yes & $77(96.2)$ & I55(96.9) \\
\hline No & $3(3.8)$ & $5(3.1)$ \\
\hline \multicolumn{3}{|c|}{$\begin{array}{l}\text { How many times have you } \\
\text { eaten vegetables }\end{array}$} \\
\hline Daily/week & $18(23.4)$ & $47(30.3)$ \\
\hline 2-3times/week & $55(71.4)$ & $100(64.5)$ \\
\hline Less than 2 times/week & $4(5.2)$ & $8(5.2)$ \\
\hline \multicolumn{3}{|c|}{$\begin{array}{l}\text { Have you eaten meat and } \\
\text { animal products }\end{array}$} \\
\hline Yes & $69(86.2)$ & I37(85.6) \\
\hline No & $\mathrm{II}(13.8)$ & $23(14.4)$ \\
\hline \multicolumn{3}{|l|}{$\begin{array}{l}\text { How many times you ate } \\
\text { animal product }\end{array}$} \\
\hline Daily/week & $5(7.5)$ & II (8.0) \\
\hline 2-3 times/week & $43(62.3)$ & $98(71.5)$ \\
\hline Less than2 times/week & $21(30.4)$ & $28(20.4)$ \\
\hline \multicolumn{3}{|l|}{$\begin{array}{l}\text { Iron foliate intake during } \\
\text { pregnancy }\end{array}$} \\
\hline Yes & $66(82.5)$ & $145(90.6)$ \\
\hline No & $14(17.5)$ & $15(9.4)$ \\
\hline \multicolumn{3}{|l|}{$\begin{array}{l}\text { Coffee drink during } \\
\text { pregnancy }\end{array}$} \\
\hline Yes & $67(83.8)$ & $137(85.6)$ \\
\hline No & $13(16.2)$ & $23(14.4)$ \\
\hline \multicolumn{3}{|l|}{$\begin{array}{l}\text { How many times did you } \\
\text { drink coffee }\end{array}$} \\
\hline Daily/week & $38(56.7)$ & $72(52.9)$ \\
\hline 2-3 times/week & $26(38.8)$ & $59(43.4)$ \\
\hline Less than 2 times/week & $3(4.5)$ & $5(3.6)$ \\
\hline
\end{tabular}

There was no history of cigarette smoking during pregnancy among both groups. Related to physical exercise $7(8.8 \%)$ of cases and $34(21.2 \%)$ of controls had practiced it. Five $(6.2 \%)$ of cases and $8(5.0 \%)$ of controls drank alcohol during pregnancy (Table 3).

\section{Factors Associated with Preeclampsia}

On bi-variable analysis, the variables like age between 20 and 34 years, attending education, marital status, place of residence, occupation, number of pregnancies, number of ANC (antenatal care) visits, having a history of abortion, types of contraceptive method, having hypertension, diabetes mellitus (DM), preeclampsia in the patient's family, having eaten fruit during pregnancy, iron folate intake during pregnancy, and practicing exercise were eligible for the final model.

On multivariable analysis the variables including attending education, occupation (housewife, having private work), age between 20 and 34 years, family history of diabetes mellitus, hypertension, preeclampsia, and having eaten fruit during pregnancy were significantly associated with preeclampsia at $P$-value less than 0.05 .

Mothers whose age is between 20 and 34 years were $95.1 \%$ less likely to acquire preeclampsia compared to age less than 20 years [AOR $=0.49,95 \%$ CI $(0.006,0.398)]$. Mothers who can read and write were $87 \%$ less likely to have preeclampsia as compared to mothers who cannot read and write $[\mathrm{AOR}=0.13,95 \% \mathrm{CI}(0.02,0.76)]$. Mothers who attended primary education were $93.1 \%$ less likely to develop preeclampsia as compared to those who cannot read and write $[\mathrm{AOR}=0.071,95 \% \mathrm{CI}(0.015,0.32)]$.

Participant housewives were 13 times more likely to have preeclampsia compared to those who were government employed [AOR $=13,95 \%$ CI $(1.260,140.15)]$. Merchant mothers were 14 times more likely to develop preeclampsia as compared to those who are government employed $[\mathrm{AOR}=14,95 \%$ CI $(1.46,137.72)]$. Mothers who have no family history of diabetes mellitus had $72.8 \%$ reduced risk of developing preeclampsia as compared to those who have a family history of diabetes mellitus [AOR $=0.28 .2,95 \%$ CI $(0.081,0.985)]$.

Mothers who have no family history of hypertension had $87.6 \%$ reduced risk of preeclampsia as compared to those who have a family history of hypertension $[\mathrm{AOR}=0.124,95 \% \mathrm{CI}$ $(0.047,0.325)]$. Mothers who have no maternal history of preeclampsia had $83.8 \%$ reduced risk of developing preeclampsia than those who have a maternal history of hypertension $[\mathrm{AOR}=0.16295 \% \mathrm{CI}(0.041,0.640)]$. Mothers who did not eat fruit during pregnancy were 3 times more likely to develop preeclampsia than those who had eaten fruit during pregnancy $[\mathrm{AOR}=3.355,95 \% \mathrm{CI}(1.112,10.126)]$. (Table 4) 
Table 4 The Bi-Variable and Multivariable Analysis Result of the Determinants of Preeclampsia Among Mothers Attending ANC, and Delivery Services in Gedeo Zone Hospitals, South Ethiopia, 2019 ( $n=240$ (80 Cases, 160 Controls))

\begin{tabular}{|c|c|c|c|c|}
\hline \multirow[t]{2}{*}{ Variables } & \multicolumn{2}{|l|}{ Study subject(240) } & \multirow[t]{2}{*}{ COR $(95 \% \mathrm{Cl})$} & \multirow[t]{2}{*}{ AOR $(95 \% \mathrm{Cl})$} \\
\hline & $\begin{array}{l}80 \text { Cases = number } \\
(\%)\end{array}$ & $\begin{array}{l}\text { I } 60 \text { Controls number } \\
\text { (\%) }\end{array}$ & & \\
\hline \multicolumn{5}{|l|}{ Age } \\
\hline$<20$ years & $10(12.5)$ & $15(9.5)$ & 1 & I \\
\hline 20-34 years & $62(77.2)$ & $125(79.1)$ & $.66(.21,2.11)$ & $.049(.006, .398)^{*}$ \\
\hline$>35$ years & $8(10.0)$ & $18(\mid I .4)$ & $.89(.36,2.17)$ & $.314(.063,1.556)$ \\
\hline \multicolumn{5}{|l|}{ Marital status } \\
\hline Single & $3(3.8)$ & $7(4.4)$ & 1 & 1 \\
\hline Married & $72(90.9)$ & $|5|(94.4)$ & $5.83(.696,48.87)$ & $1.65(.134,20.233)$ \\
\hline Divorced & $5(6.2)$ & $2(1.2)$ & $5.24(.99,27.67)$ & $2.13(.31,14.425)$ \\
\hline \multicolumn{5}{|l|}{ Educational status } \\
\hline Cannot read and write & $28(35.0)$ & $46(28.8)$ & 1 & 1 \\
\hline Can read and write & $28(35.0)$ & $30(18.8)$ & $.430(.191, .970)$ & $.13(.02, .76)^{*}$ \\
\hline Primary education & $5(6.2)$ & $23(14.4)$ & $.281(.121, .650)$ & $.07 \mid(.015, .32 *$ \\
\hline Secondary education & $8(10.0)$ & $19(\mid 1.9)$ & $1.205(.373,3.894)$ & $.88(.12,6.25)$ \\
\hline College and above & $\mathrm{II}(\mathrm{I3.8)}$ & $42(26.2)$ & $.622(.216,1.795)$ & $.23(.05,1.028)$ \\
\hline \multicolumn{5}{|l|}{ Residences } \\
\hline Urban & $30(37.5)$ & $47(29.4)$ & 1 & 1 \\
\hline Rural & $50(62.5)$ & $113(70.6)$ & $.693(.394,1.221)$ & $.590(.193,1.806)$ \\
\hline \multicolumn{5}{|l|}{ Occupation } \\
\hline Government employee & $20(25.0)$ & $47(29.4)$ & I & I \\
\hline Private & $9(11.2)$ & $22(13.8)$ & $1.95(.535,7.165$ & $3(.355,25.760)$ \\
\hline Housewife & $30(37.5)$ & $60(37.5)$ & $2.037(.493,8.408)$ & $13(1.260,140.15)^{*}$ \\
\hline Merchant & $16(20.0)$ & $25(15.6)$ & I.667(.470, 5.906) & $14(1.46,137.72)^{*}$ \\
\hline others & $5(6.2)$ & $6(3.8)$ & $1.302(.340,4.985)$ & $8(.802,80.68)$ \\
\hline \multicolumn{5}{|l|}{ Monthly Income } \\
\hline $570-1140$ & $5(9.3)$ & $14(|| .4)$ & I & I \\
\hline$\geq 1140$ & $49(90.7)$ & $109(88.6)$ & $1.25(.429,3.689)$ & $1.845(.452,7.53)$ \\
\hline \multicolumn{5}{|l|}{ Number of pregnancies } \\
\hline One & $\mid 7(2 \mid .2)$ & $53(35.5)$ & 1 & I \\
\hline $2-4$ & $5 \mathrm{I}(63.8)$ & $86(54.4)$ & $1.969(.796,4.872)$ & $1.002(.207,4.850)$ \\
\hline$\geq 5$ & $12(15.0)$ & $19(12.0)$ & I.065 (.478, 2.374) & $.586(.156,2.210)$ \\
\hline \multicolumn{5}{|l|}{ The number of ANC follow-ups } \\
\hline One time & $4(5.5)$ & $8(5.3)$ & 1 & $\mathrm{I}$ \\
\hline Two times & $19(26.0)$ & $28(18.7)$ & $.655(.176,2.433)$ & $.365(.062,2.162)$ \\
\hline Three times & $32(43.8)$ & $59(39.3)$ & $.482(.219,1.062)$ & $.525(.155,1.783)$ \\
\hline Four and above & $18(24.7)$ & $55(36.7)$ & $.603(.304,1.196)$ & $.420(.139,1.270)$ \\
\hline \multicolumn{5}{|l|}{ History of abortion } \\
\hline Yes & $12(15.0)$ & $19(11.9)$ & I & I \\
\hline No & $68(85.0)$ & $|4|(88.1)$ & $.764(.35 \mathrm{I}, \mathrm{I} .663)$ & I.272(.444, 3.645) \\
\hline \multicolumn{5}{|l|}{ Type of contraceptive method used } \\
\hline Pills & $4(6.2)$ & $13(10.9)$ & $\mathrm{I}$ & 1 \\
\hline Inject able & $36(56.2)$ & $67(56.3)$ & $2.00(.584,6.847)$ & $5.605(.823,38.167)$ \\
\hline Implant & $23(35.9)$ & $39(32.8)$ & I.I45 (.59, 2.194) & I.194(.486, 2.935) \\
\hline
\end{tabular}

(Continued) 
Table 4 (Continued).

\begin{tabular}{|c|c|c|c|c|}
\hline \multirow[t]{2}{*}{ Variables } & \multicolumn{2}{|l|}{ Study subject(240) } & \multirow[t]{2}{*}{ COR $(95 \% \mathrm{Cl})$} & \multirow[t]{2}{*}{ AOR $(95 \% \mathrm{Cl})$} \\
\hline & $\begin{array}{l}80 \text { Cases = number } \\
\text { (\%) }\end{array}$ & $\begin{array}{l}\text { I60 Controls number } \\
\text { (\%) }\end{array}$ & & \\
\hline \multicolumn{5}{|l|}{ Family history of diabetes mellitus } \\
\hline Yes & $\mathrm{II}(\mathrm{I} .8)$ & $6(3.8)$ & I & I \\
\hline No & $69(86.2)$ & $154(96.2)$ & $.244(.087, .688)$ & $.282(.081, .985)^{*}$ \\
\hline \multicolumn{5}{|l|}{ Family history of hypertension } \\
\hline Yes & $26(32.5)$ & $8(5.0)$ & 1 & I \\
\hline No & $54(67.5)$ & $152(95.0)$ & $.109(.047, .256)$ & $.124(.047, .325)^{*}$ \\
\hline \multicolumn{5}{|l|}{ Family history of hypertension } \\
\hline Yes & $26(32.5)$ & $8(5.0)$ & I & I \\
\hline No & $54(67.5)$ & $152(95.0)$ & $.109(.047, .256)$ & $.124(.047, .325)^{*}$ \\
\hline \multicolumn{5}{|l|}{$\begin{array}{l}\text { Participants' maternal history of } \\
\text { preeclampsia }\end{array}$} \\
\hline Yes & $16(20.0)$ & $4(2.5)$ & 1 & I \\
\hline No & $64(80.0)$ & $156(97.5)$ & $.103(.033, .319$ & $.162(.041, .640)^{*}$ \\
\hline \multicolumn{5}{|l|}{ Presence of diabetes mellitus } \\
\hline Yes & $6(7.5)$ & $6(3.8)$ & 1 & 1 \\
\hline No & $74(92.5)$ & I54(96.2) & $.48 I(.150,1.54 I)$ & $.27 I(.053,1.389)$ \\
\hline \multicolumn{5}{|l|}{ Ate Fruit during pregnancy } \\
\hline Yes & $7 I(88.8)$ & $150(93.8)$ & 1 & I \\
\hline No & $9(11.2)$ & $10(6.2)$ & $1.901(.740,4.886)$ & $3.355(1.112,10.126)^{*}$ \\
\hline \multicolumn{5}{|l|}{ Iron foliate intake during pregnancy } \\
\hline Yes & $66(82.5)$ & $145(90.6)$ & I & 1 \\
\hline No & $14(17.5)$ & $15(9.4)$ & $2.051(.936,4.493)$ & $2.59(.975,6.89)$ \\
\hline \multicolumn{5}{|l|}{ Physical exercise during pregnancy } \\
\hline Yes & $7(8.8)$ & $34(21.2)$ & I & 1 \\
\hline No & $73(9 \mid .2)$ & $126(78.8)$ & $2.814(1.187,6.670)$ & $2.537(.945,6.810)$ \\
\hline
\end{tabular}

Note: ${ }^{*} p$-value $<0.05$

\section{Discussion}

An unmatched case-control study was conducted among 240 mothers who attended ANC and delivery services in the three hospitals. According to this study, the main determinant factors of preeclampsia are attending education, age range between 20 and 34 years, occupation, family history of hypertension, family history of diabetes mellitus, family history of preeclampsia, and eating fruit during pregnancy.

The current study showed that mothers who were between 20 and 34 years old have a $95.1 \%$ reduced risk of developing preeclampsia as compared to mothers aged less than 20 years. The finding is supported by studies in Dessie $^{16}$ and India, ${ }^{15}$ and those older than 35 are at increased risk of developing preeclampsia. This might be explained by mothers who are too young may not be physiologically mature enough to cope with the demands of pregnancy, and older mothers above 35 years are more likely to have cardiovascular diseases, which is probably reflected in an increase in the incidence of essential or latent hypertension. ${ }^{16}$ This might be also due to the steady decrement of elasticity of the blood vessels mainly related to aging and arterial stiffness. Additionally, when mothers get older, hemodynamic maintenance during pregnancy and delivery becomes more difficult. ${ }^{16}$

The mothers who attended primary education had a $92.9 \%$ reduced risk of getting preeclampsia than the mothers who cannot read and write. This result is supported by a study done in the Gaza Strip ${ }^{17}$ and Jakarta. ${ }^{18}$ This could be explained by uneducated mothers having reduced access to early prevention and control 
mechanisms; healthy nutrition, avoiding sedentary life, and prevention of overweight and obesity ${ }^{19}$ are identified risk factors for preeclampsia. Mothers who have low educational level had $86 \%$ higher risk of preeclampsia, while middle education level had a $72 \%$ higher risk of preeclampsia than those who have higher educational level. ${ }^{18}$

The current study revealed that housewives were 13 times more likely to develop preeclampsia than government-employed mothers. This result is supported by a study done in Jahun, Nigeria. ${ }^{18}$ This might be due to low educational attainment, which may result in increased anxiety and depression. Anxiety and depression during early gestation are related to the risk of preeclampsia. In contrast to this, working mothers outside the home are at higher risk of acquiring preeclampsia than housewife mothers. ${ }^{20}$ Working mothers are more prone to develop $\mathrm{PE}$ as they are more stressed (in terms of traveling during pregnancy and job responsibility) than housewives. This study explained that merchant mothers were 14 times more likely to develop preeclampsia than government-employed mothers. This might be due to the high-stress situation of the work activity.

In the current finding, mothers who have no family history of diabetes mellitus had a $72.8 \%$ reduced risk of getting preeclampsia than those who have a family history of diabetes mellitus. This is supported by studies done in Dessie Hospital ${ }^{16}$ and Jaipur India. The likelihood of preeclampsia in Dessie and India was nearly increased by 2.4 and 2.3 times if diabetes is present before pregnancy, respectively. Diabetes is a significant risk factor for preeclampsia which exposed the mothers to poor glycemic control and obesity; this in turn increases the deposition of fats under blood vessels, which results in an increased risk of hemodynamic instability.

The current finding revealed that mothers who have no family history of hypertension had $87.6 \%$ reduced risk of developing preeclampsia than those who have a family history of hypertension. The finding is supported by studies done in Dessie Hospital ${ }^{16}$ and India, ${ }^{15}$ which revealed that the mother's family hypertension was associated with preeclampsia cases, compared with normotensive controls identified at delivery. This might be because susceptibility to preeclampsia is highly heritable and population-based studies have shown a strong familial tendency to preeclampsia. ${ }^{21}$

This study showed out that mothers who have no family (maternal) history of preeclampsia had $83.8 \%$ reduced risk of developing preeclampsia than those who have a maternal history of hypertension. Other findings in Dessie $^{16}$ and India ${ }^{22}$ support this result, as mothers who have a family history of hypertension are more likely to develop preeclampsia than their counterparts. This could be explained by the probability of acquiring the risk factors of preeclampsia being transferred from parents through genetic factors.

The current study showed that mothers who did not eat fruit during pregnancy were 3 times more likely to develop preeclampsia than those who had eaten fruit during pregnancy. A study done in northwest Ethiopia revealed that folate supplementation during pregnancy and fruit feeding was related to decreased risk of preeclampsia. ${ }^{23}$ This might be due to the micronutrients available in fruits such as vitamins, minerals, antioxidants, and dietary fibers, which are known to reduce the risk of hyper-homocysteinemia, identified risk factors for preeclampsia. ${ }^{24}$

\section{Conclusion and Recommendation}

The study identified that the determinant factors for preeclampsia include ability to read and write, primary education, maternal age between 20 and 34 years, occupation (housewife, having private work), positive family history of hypertension, diabetes mellitus, maternal history of preeclampsia, and eating fruits during pregnancy. Strengthening early detection and prevention of predisposing factors, and improving protective factors, such as educating mothers in collaboration with the education sector, are recommended.

\section{Ethical Standard of Disclosure}

This study was conducted in accordance with the Declaration of Helsinki. The study was approved by the ethical review board (IRB) of the college of health science and medicine, Dilla University. The participants were informed about the purpose of the study and the names of the respondents were not recorded to maintain confidentiality. The study participants were also given the right to discontinue participation at any time during the interview. Finally, data were collected after obtaining written informed consent from the study subjects.

\section{Acknowledgments}

We would like to express our heartfelt gratitude to Dilla University for ethical clearance. Our gratefulness also extends to the staff of the Gedeo Zone health department for their collaboration. Last but not least, we would like to acknowledge our data collectors, supervisors, and all study 
participants who spent their productive time for the completion of this study.

\section{Author Contributions}

All authors made substantial contributions to conception and design, acquisition of data, or analysis and interpretation of data; took part in drafting the article or revising it critically for important intellectual content; agreed to submit to the current journal; gave final approval of the version to be published; and agree to be accountable for all aspects of the work.

\section{Disclosure}

The authors declared that they have no competing interests in this work.

\section{References}

1. for the Revision IAG. A conceptual framework for the revision of the ICD-10 classification of mental and behavioural disorders. World Psychiatry. 2011;10(2):86. doi:10.1002/j.2051-5545.2011.tb00022.x

2. Sibai B, Dekker G, Kupferminc M. Pre-eclampsia. Lancet. 2005;365 (9461):785-799. doi:10.1016/S0140-6736(05)17987-2

3. Duckitt K, Harrington D. Risk factors for pre-eclampsia at antenatal booking: systematic review of controlled studies. BMJ. 2005;330 (7491):565. doi:10.1136/bmj.38380.674340.E0

4. Organization WH. Health Guidelines for the Use of Wastewater in Agriculture and Aquaculture. Report of a WHO Scientific Group [Meeting Held in Geneva from 18 to 23 November 1987]. World Health Organization; 1989.

5. Giannubilo SR, Cecchi S, Tidu E, Ciavattini A. Maternal NT-proBNP in chronic hypertensive pregnancies and superimposed preeclampsia. Int $J$ Cardiol. 2014;176(3):1227-1229. doi:10.1016/j.ijcard.2014. 07.214

6. Lee CJ, Hsieh TT, Chiu TH, Chen KC, Lo LM, Hung TH. Risk factors for pre-eclampsia in an Asian population. Int $J$ Gynecol Obstet. 2000;70(3):327-333. doi:10.1016/S0020-7292(00)00240-X

7. Zhang F, Dong L, Zhang C, et al. Increasing prevalence of gestational diabetes mellitus in Chinese women from 1999 to 2008. Diab Med. 2011;28(6):652-657. doi:10.1111/j.1464-5491.2010.03205.x

8. Organization WH. Trends in Maternal Mortality: 1990 to 2008. Geneva: World Health Organization; 2010.

9. Organization WH. Strategic Directions for Accelerating the Reduction of Maternal Mortality in the Eastern Mediterranean Region: A Regional Framework; 2005.
10. Osungbade KO, Ige OK. Public health perspectives of preeclampsia in developing countries: implication for health system strengthening. J Pregnancy. 2011;2011.

11. Khan KS, Wojdyla D, Say L, Gülmezoglu AM, Van Look PF. WHO analysis of causes of maternal death: a systematic review. lancet. 2006;367(9516):1066-1074. doi:10.1016/S0140-6736(06)68397-9

12. Al-Tairi ANQ, Isa ZM, Ghazi HF. Risk factors of preeclampsia: a case control study among mothers in Sana'a, Yemen. J Public Health. 2017;25(6):573-580. doi:10.1007/s10389-017-0825-0

13. Garomssa H, Dwivedi A. Maternal mortality in Ambo Hospital: a five year retrospective review. Ethiopian $j$ Reproductive Health. 2008;2:1.

14. Wolde Z, Segni H, Woldie M. Hypertensive disorders of pregnancy in Jimma University specialized hospital. Ethiop $J$ Health Sci. 2011;21:3.

15. Ganesh KS, Unnikrishnan B, Nagaraj K, Jayaram S. Determinants of pre-eclampsia: a case-control study in a district hospital in South India. Indian j Community Med. 2010;35(4):502. doi:10.4103/09700218.74360

16. Tessema GA, Tekeste A, Ayele TA. Preeclampsia and associated factors among pregnant women attending antenatal care in Dessie referral hospital, Northeast Ethiopia: a hospital-based study. $B M C$ Pregnancy Childbirth. 2015;15(1):73. doi:10.1186/s12884-0150502-7

17. Samar E. Case-control study of risk factors associated with preeclampsia in the Gaza strip. J Med Med Sci. 2015;6(9):229-233.

18. Van Katwijk C, Peeters LL. Clinical aspects of pregnancy after the age of 35 years: a review of the literature. Hum Reprod Update. 1998;4(2):185-194. doi:10.1093/humupd/4.2.185

19. Ennazhiyil SV, Valsan SM, Rajeev AV, Srinivasan C, Kunnath RP. The socio-demographic determinants of gestational diabetes mellitus among postnatal women from Palakkad district, Kerala: comparative study. Int J Community Med Public Health. 2019;6(6):2449. doi:10.18203/2394-6040.ijcmph20192303

20. Opitasari C, Andayasari L. Parity, education level and risk for (pre-) eclampsia in selected hospitals in Jakarta. Health Sci J Indonesia. 2014;5(1):35-39.

21. Ros HS, Cnattingius S, Lipworth L. Comparison of risk factors for preeclampsia and gestational hypertension in a population-based cohort study. Am J Epidemiol. 1998;147(11):1062-1070. doi:10.109 3/oxfordjournals.aje.a009400

22. Marcoux S, Brisson J, Fabia J. The effect of leisure time physical activity on the risk of pre-eclampsia and gestational hypertension. $J$ Epidemiol Community Health. 1989;43(2):147-152. doi:10.1136/ jech.43.2.147

23. Cooper AA, Chen Y-J, Lindorfer MA, Stevens TH. Protein splicing of the yeast TFP1 intervening protein sequence: a model for selfexcision. EMBO J. 1993;12(6):2575-2583. doi:10.1002/j.14602075.1993.tb05913.x

24. Endeshaw M, Abebe F, Worku S, Menber L, Assress M, Assefa M. Obesity in young age is a risk factor for preeclampsia: a facility based case-control study, northwest Ethiopia. BMC Pregnancy Childbirth. 2016;16(1):237. doi:10.1186/s12884-016-1029-2
The International Journal of Women's Health is an international, peerreviewed open-access journal publishing original research, reports, editorials, reviews and commentaries on all aspects of women's healthcare including gynecology, obstetrics, and breast cancer. The manuscript management system is completely online and includes a very quick and fair peer-review system, which is all easy to use. Visit http://www.dovepress.com/testimonials.php to read real quotes from published authors. 